that the usual rates, as obtained by the standard Kyropoulos method, could be considerably increased by undercooling the melt. Melts can be undercooled by as much as $20-30^{\circ} \mathrm{C}$. without risk of nucleation. Dr. Billig showed that if one (111) plane of a germanium crystal is maintained vertical, the crystal is able to grow without the need for nucleating new layers; no experimental evidence of growth from spiral dislocations has in fact been obtained in this work. Imperfections, however, do arise, due possibly to the occlusion of impurities and to mechanical stresses. Such stresses are commonly caused by uneven temperature distribution or by certain crystallographic features such as the intersection of two twin boundaries. A single twin boundary, on the other hand, apparently has no effect on the electrical properties. Dr. Billig concluded by showing a film illustrating various features in the growth of germanium and silicon monocrystals. Under certain conditions, such crystals have been obtained in the form of thin plates which display a striking degree of external symmetry. The film also showed the extremely rapid growth of dendritic crystals in the form of thin and long lamellæ, demonstrating in a convincing manner that, even in an isotropic medium of cubic symmetry, crystal growth can take place by the deposition of individual layers orientated along the close-packed (111) planes.

F. S. STONE

\section{STAR-STREAMING FROM STARS OF SPECTRAL TYPES A AND B}

A PAPER by T. R. Tannahill on "Star-Streaming A from the Stars of Spectral Type B" (Mon. Not. Roy. Astro. Soc., 114, No. 4 ; 1954) gives the results of his analysis, on Eddington's two-drift hypothesis, of the proper motions of 1,536 stars of spectral types $O$ and $B$ in the Boss "General Catalogue". The material for the investigation was grouped into ten regions, each region consisting of a 'square' of north galactic latitude combined with the diametrically opposite region of south galactic latitude, as shown in the first table of the paper. An examination of this table and also of the drift-curves establishes that it is impossible to represent the motions of the $B$-type stars by a single drift, and a large proportion of the stars has been assigned to a second drift in order to make a satisfactory fit; in four out of the ten regions in Drift ii the number of stars exceeds that in Drift i. From an analysis of the data, the drift constants are derived with the probable errors.

In a previous paper (Mon. Not. Roy. Astro. Soc., 112 , No. 3 ; 1952), Tannahill gave the corresponding results from the galactic zone of stars of types $A_{5}-M$, and a comparison with the present results indicates satisfactory accordance, considering the probable errors involved. The values of $L, B$ and $h W$, the galactic co-ordinates of each drift apex and the space velocities of each drift, are used to calculate the theoretical values of $h V_{c}$ and $\theta_{c}$-that is, the projections of the space-velocities of the drifts on the tangent-plane at the centre of the region, and the position angles of the directions towards the drift apices-and these are listed together with the residuals $h V_{c}-h V$ and $\theta_{c}-\theta$. Considering that only 1,536 stars were used in the investigation, the residuals are quite satisfactory except in Drift $i$, region 5 , where $\theta_{c}-\theta_{1}$ exceeds $49^{\circ}$. The linear components of the motion of Drift i relative to Drift ii are given in a table, and also the galactic longitude and latitude of the vertex of star-streaming and the relative spacevelocity of the drifts. One curious feature is the close agreement of the galactic co-ordinates of the vertex position $\left(343 \cdot 3^{\circ},-1 \cdot 6^{\circ}\right)$ obtained by the investigation, and that normally found $\left(343^{\circ},-2^{\circ}\right)$. It is admitted that the closeness of the agreement must be regarded as fortuitous, on account of the probable errors involved. Nevertheless, this does not vitiate the final conclusion, and the result "removes any shadow of doubt that the $B$-type stars exhibit the phenomenon of star-streaming".

The subject is continued in a further paper (Mon. Not. Roy. Astro. Soc., 114, No. 5 ; 1954), "StarStreaming from the Proper Motions of the $A$-Type Stars of the Boss 'General Catalogue'", in which are given the results of the analysis of the motions of 8,676 stars of the spectral types $B_{8}-A_{3}$. The method of dealing with the data follows closely that described in the 1952 paper for stars of types $A_{5}-M$, and stars belonging to known clusters have been rejected; of these, twenty belong to the Pleiades group, forty-six to the Ursa Major group, and nine to the Taurid cluster. The same difficulty in analysis arose as with the $A_{5}-M$ stars, the regions around Drift i apex offering certain problems in analysis, especially in the determination of the Drift ii constants. The higher concentration of the stars in the galactic plane, the regions in the higher galactic latitudes being sparsely populated, presented further difficulties; but in spite of these, analysis was possible in all regions, and the results are given in nine tables.

One rather surprising feature may be mentioned : in the $A_{5}-M$ types there was a marked decrease of $h W$ with advancing spectral type, which was not maintained in the $A$-stars. There was a decided systematic change in the Drift ii apex, the right ascension decreasing, and the declination increasing, with advancing type. In addition, large and apparently irregular variations occurred in some of the quantities determined, and, judging by the probable errors involved, must have a statistical significance, yet they indicated no systematic trend. Some possible reasons for these discrepancies are suggested by Tannahill, and though they are only tentative, it is probable that future researeh will modify some of the hypotheses on which present investigations are based. The galactic co-ordinates of the vertex were found to be $\left(365.5^{\circ},-0.5^{\circ}\right)$, and the equatorial co-ordinates of the solar apex $\left(264 \cdot 8^{\circ},+31 \cdot 5^{\circ}\right)$; the latter is not far from the co-ordinates of the solar apex as found from the streaming of the spectral type $B$ stars $\left(260 \cdot 7^{\circ}+37 \cdot 3^{\circ}\right)$.

\section{THE INSTITUTE OF SEAWEED RESEARCH}

TN the annual report for 1954 of the Institute of 1 Seaweed Research, the interested layman's attention will be attracted by the statement that "mechanical harvesting of sublittoral Laminariaceae has now been proved to be a feasible and reasonably cheap operation". At present, six seaweed-processing factories in Scotland use more than 30,000 tons of cast weed annually, and it is estimated that one motor fishing vessel adapted to operate twin I.S.R. belt harvesters should be able to collect more than five thousand tons of Laminaria cloustoni a year in good areas. One of the main objectives of the Institute 
has therefore been attained and the advantage to the seaweed industry of planned collection of the material over cast weed is obvious.

If mechanical harvesting has been the limiting factor in the industry up to the present, its adoption may bring fresh problems. If it becomes regular practice, limited in the main by the state of the weather and sea only-it is suggested that harvesting on at least 170 days a year is a reasonable assumption-it becomes important to assess the effects of harvesting on the density of the beds of Laminaria cloustoni as well as on the associated fauna and flora. The same report indicates that changes in the density of these beds occur from year to year when they are subject to natural conditions only, and henceit is reason able to ask what effect harvesting will have on them.

Processes for the manufacture of mannitol, aminarin, calcium alginate and fucosterol have been perfected at the Institute; but whatever products are to be made use of commercially, the industry is in the last resort dependent on a continuing supply of the plant. Experimental work on the effect of harvesting is therefore of prime importance.

Kathleen M. DReW

\section{WORK STUDY}

$\mathrm{I}^{\mathrm{N}}$ one of the occasional papers published by the Institute of Personnel Management, a useful corrective is given to the unfortunate trend in industry which regards work study as the panacea for all industrial ills. Valuable as they are, work study and monetary incentive schemes are techniques which can have the most harmful consequences; they may increase production in the short run (not always in the long run) but at a social and psychological cost which is no less serious because it cannot be immediately perceived. It may be argued that it is unjustifiable to criticize work study together with the use of narrowly conceived incentive schemes, on the ground that there is no necessary connexion between them. There is, of course, no necessary connexion, but, in practice, there has developed a very natural connexion: the increase in repetitive work and the progressive simplification of the job have required that a substitute incentive be found for the satisfaction and skill so often destroyed. Among the many dangers and disadvantages of direct incentive schemes the following seem to be among the most important.

They are sought and used as a substitute for managerial skill in other directions, and they tend to deflect attention from matters which should have a far higher degree of priority in the development of any management policy; for example, production planning and sales/production co-ordination, educa. tion and training, method study, and systems of cost control.

The foreman's direct responsibility for output is reduced; this is said to be an advantage in that he is thus enabled to carry out more important duties. In fact, the stresses impinging on the foreman are frequently increased by the introduction of an incentive scheme and become more frustrating and harmful in character.

The impact of incentive earnings on the wages structure can be very detrimental and lead to much discontent. Supervisory differentials and other evaluated jobs are adversely affected by distorted earnings in other departments.
The installation of an individual incentive scheme frequently has a disintegrating effect on the working group which in many cases is resolved by tacit agreement among workers to restrict output to an agreed level ; this is strongly condemned by management, who fail to understand that in the circumstances it is indispensable for the social equilibrium of the group.

As the whole emphasis of the incentive scheme is on producing the maximum individual reward, the effect is for the incentive bonus to be won regardless of necessary co-operation with supervisors and fellowworkers. The quality of production frequently suffers despite careful inspection procedures.

The scientific accuracy of time study has yet to be demonstrated, and the trade unions, aware of the difference in standards between time-study men, are not slow to take advantage of these opportunities in negotiation; in this respect time study may be regarded as a tool for collective bargaining.

Not the least important is the insidious effect that incentive schemes can have on the moral qualities and sense of responsibility of the individual. It is perhaps to be expected that a system which attempts to allocate a precisely defined reward for every action of the individual in his working socioty should have its repereussions elsewhere.

\section{CONGENITAL PORPHYRIA IN SWINE AND CATTLE IN DENMARK}

\author{
By S. K. JØRGENSEN \\ Thisted Slaughter-House, Denmark \\ AND \\ DR. TORBEN K. WITH
}

Central Laboratory, County Hospital, Svendborg, Denmark CONGENITAL porphyria is a rare inborn error of $\checkmark$ metabolism in man and animals. It is occasionally seen in pigs and cattle in slaughter-houses because of the reddish-brown colour of the bones and teeth; but it has only once been studied in live cattle ${ }^{1}$ and live pigs $^{2}$. For further information the reader is referred to the survey by Rimington ${ }^{2}$. It is a puzzling coincidence that congenital porphyria has been found in both pigs and cattle in Thisted County in North Jutland. We are now studying the live animals.

In October 1951 one of us (S. K. J.) found dark bones in pigs at Thisted slaughter-house. This reduced the market value of the pork, and in consequence an investigation was started. The condition could be diagnosed with reasonable certainty on inspection of the colour of the teeth in the newborn pigs; pigs with dark teeth as a rule develop porphyria. After elimination of all pigs with dark teeth, the condition disappeared; since February 1954 no new cases have occurred at Thisted. Two sows with porphyria were transferred to the State Experiment Farm of Faurholm for breeding studies and chemical investigations under the direction of Prof. H. Clausen and a senior experiment officer, N. J. Højgaard Olsen, in collaboration with one of us (T. W.). Some material was also sent to Prof. C. Rimington in London.

The best method of characterizing the severity of porphyria in a pig is quantitative analysis of the 